\title{
Estado de conservación del jaguar y el pecarí de labio blanco en el Ecuador occidental
}

\section{Conservation status of the jaguar and the white-lipped peccary in western Ecuador}

\author{
Galo Zapata-Ríos ${ }^{1,2}$, Edison Araguillin ${ }^{1,3}$
}

\begin{abstract}
Resumen
Poco se conoce sobre el estado de conservación de las poblaciones de jaguar y pecarí de labio blanco al occidente de los Andes en el Ecuador. Muestreamos las dos especies en los cuatro remanentes de bosque más grandes del Ecuador occidental, utilizando entrevistas a la gente local, senderos de reconocimiento y trampas fotográficas. Las dos especies fueron detectadas únicamente en uno de los remanentes (Reserva Ecológica Cotacachi-Cayapas y su zona de amortiguamiento un área de 2500 $\mathrm{km}^{2}$ ). Aparentemente, ambas especies han sido ya extirpadas de los otros tres remanentes grandes de bosque en la región y su persistencia a largo plazo depende de acciones de conservación inmediatas en la Reserva Ecológica Cotacachi-Cayapas.
\end{abstract}

Palabras clave: Carnívoros, Extinción local, Muestreos de reconocimiento, Trampas fotográficas, Ungulados.

\begin{abstract}
Little is known about the conservation status of jaguar and white-lipped peccary populations west of the Andes in Ecuador. We surveyed the two species in the four largest forest remnants in western Ecuador, using interviews to local people, reconnaissance surveys, and camera traps. The two species were detected only in one of the remnants (Cotacachi-Cayapas Ecological Reserve and its buffer zone, an area of $2,500 \mathrm{~km}^{2}$ ). It appears, both species have been extirpated already from the other three large forest remnants in the region, and their long-term persistence depends on immediate conservation actions in Cotacachi-Cayapas Ecological Reserve.
\end{abstract}

Keywords: Carnivores, Camera traps, Local extinction, Reconnaissance surveys, Ungulates.

\section{Introducción}

En Ecuador, el jaguar (Panthera onca) y el pecarí de labio blanco (Tayassu pecari) presentan una distribución disyunta a ambos lados de los Andes: en la Amazonía y la Costa del Pacífico (Sanderson et al. 2002, Tirira 2007, Taber et al. 2008). Las poblaciones de las dos especies, a ambos lados de la cordillera, se encuentran aisladas geográficamente unas de otras (Tirira 2007) y aunque los efectos negativos de las actividades humanas (e.g. cacería, pérdida de hábitat) amenazan las poblaciones de ambas espe- cies en la Amazonía, ninguna de las dos se encuentra en inminente riesgo de extinción. Por el contrario, las poblaciones de la Costa, en el Ecuador occidental, están desapareciendo rápidamente en todo su ámbito de distribución geográfica y poco se conoce sobre su estado de conservación actual (Tirira 2007, 2011).

La desaparición de ambas especies en la Costa ecuatoriana tendría impactos ecológicos graves. Es enorme la magnitud de los roles tróficos y ecológicos del jaguar y el pecarí de labio blanco en el mantenimiento de la estructura y funcionamiento de los

Wildlife Conservation Society.Quito, Ecuador. e-mail: gzapata@wcs.org

Department of Wildlife Ecology and Conservation, University of Florida, Gainesville, USA.

Departamento de Ecología, Universidad Autónoma de Madrid, Madrid, España. e-mail: earaguillin@wcs.org

Fecha recepción: Abril 29, $2013 \quad$ Fecha aprobación: Mayo 30, 2013 
ecosistemas que habitan. En el caso del jaguar, la extirpación de los depredadores grandes de un ecosistema produce una serie de efectos cascada por la falta de regulación demográfica de las especies presa (Terborgh 1988, Dirzo y Miranda 1990, Terborgh et al. 1999, Dirzo y Mendoza 2007, Jorge et al. 2013). Los pecaríes de labio blanco, por su parte, son considerados «ingenieros de los ecosistemas» (Jones et al. 1994, Taber et al. 2008, Altrichter et al. 2011), y juegan un papel irremplazable en la dinámica de regeneración del suelo del bosque, la dispersión de semillas y en la formación de saladeros (Painter y Rumiz 1999, Fragoso 2005, Beck 2006, Keuroghlian y Eaton 2008, 2009).

En este artículo reportamos los resultados de una serie de muestreos para ambas especies realizados en los cuatro remanentes de bosque más grandes del Ecuador occidental. El objetivo de estos muestreos fue determinar la distribución geográfica y el tamaño de la población de ambas especies. La Costa ecuatoriana es una región gravemente amenazada por la destrucción del hábitat, por lo que esta información es esencial para planificar estrategias de conservación que garanticen la conservación a largo plazo de jaguares, pecaríes de labio blanco, y otras especies amenazadas.

\section{Metodología}

Área de estudio. En la Costa ecuatoriana, un área de aproximadamente $70.000 \mathrm{~km}^{2}$, hace menos de un siglo (ca. 1938) existían aproximadamente 60.000 $\mathrm{km}^{2}$ de bosque, incluyendo bosques secos, húmedos y pluviales (Dodson y Gentry 1991). Esta gran variedad de hábitats incluye, entre otros, al norte los bosques pluviales de la sección meridional del Chocó Biogeográfico y los bosques secos de la sección septentrional de la Región Tumbesina al sur. Ambas regiones, Chocó y Tumbesina, están incluidas dentro de uno de los hotspots en términos de biodiversidad (Tumbes-Chocó-Magdalena) y están consideradas ecoregiones de importancia global (Myers 1988, Dinerstein et al. 1995, Mittermeier et al. 1998, Myers et al. 2000).

Los bosques del Ecuador occidental han sido citados como una de las áreas más amenazadas del planeta en términos de extinción biológica como resultado principalmente de procesos de destrucción del hábitat a gran escala (Myers 1988, Dodson y Gentry 1991, Brooks et al. 2002). Para finales del siglo XX, la superficie de bosques nativos se había reducido en más de un $90 \%$ por causa del crecimiento poblacional, la tala de madera y la expansión de la frontera agrícola. Como resultado de estos niveles de deforestación, los remanentes de bosque actualmente son muy pequeños y aislados, y existen muy pocas áreas de bosque nativo mayores a 500 $\mathrm{km}^{2}$ (Parker III y Carr 1992, Sierra y Stallings 1998, Sierra 1999a, Sierra et al. 2002).

Métodos de muestreo. Para determinar la presencia de jaguar y pecarí de labio blanco, realizamos una serie de muestreos de ambas especies en los principales remanentes de bosque del Ecuador occidental $\left(>500 \mathrm{~km}^{2}\right)$. Utilizando un mapa de vegetación remanente del Ecuador (Sierra et al. 2002) seleccionamos los remanentes de bosque más grandes de la región, los cuales representan cuatro áreas de estudio independientes (distanciadas por al menos $50 \mathrm{~km}$ entre una y otra). Los cuatro remanentes se caracterizan por contener áreas continuas de bosque nativo $>500 \mathrm{~km}^{2}$, donde el tipo de vegetación dominante es el bosque húmedo tropical (Sierra 1999b). En términos de tenencia de la tierra, los remanentes se encuentran dentro del sistema nacional de áreas protegidas, reservas comunitarias y privadas, y territorios indígenas. Los remanentes incluyen: 1. La Reserva Étnica Awá, 2. La Reserva Ecológica Mache-Chindul, 3. El Bosque Protector Chongón-Colonche, 4. La Reserva Ecológica Cotacachi-Cayapas y su área de amortiguamiento.

Con base en exploraciones en el campo, donde constatamos que existían áreas con hábitat disponible en los cuatro remanentes, seleccionamos de forma aleatoria nueve localidades de muestreo de aproximadamente $50 \mathrm{~km}^{2}$ cada una (Tabla 1, Figura 1). En estas nueve localidades realizamos muestreos de pecaríes y jaguares entre agosto de 2009 y agosto de 2011. Los muestreos incluyeron entrevistas a cazadores, trampeo fotográfico y senderos de reconocimiento. La duración de los muestreos en cada una de las nueve localidades varió entre 29 y 39 días efectivos de trabajo en el campo. Todas las localidades muestreadas presentaron un rango altitudinal que varió entre 200 y $900 \mathrm{~m}$.

Las entrevistas se diseñaron para evaluar la presencia de jaguares y pecaríes en las localidades de 


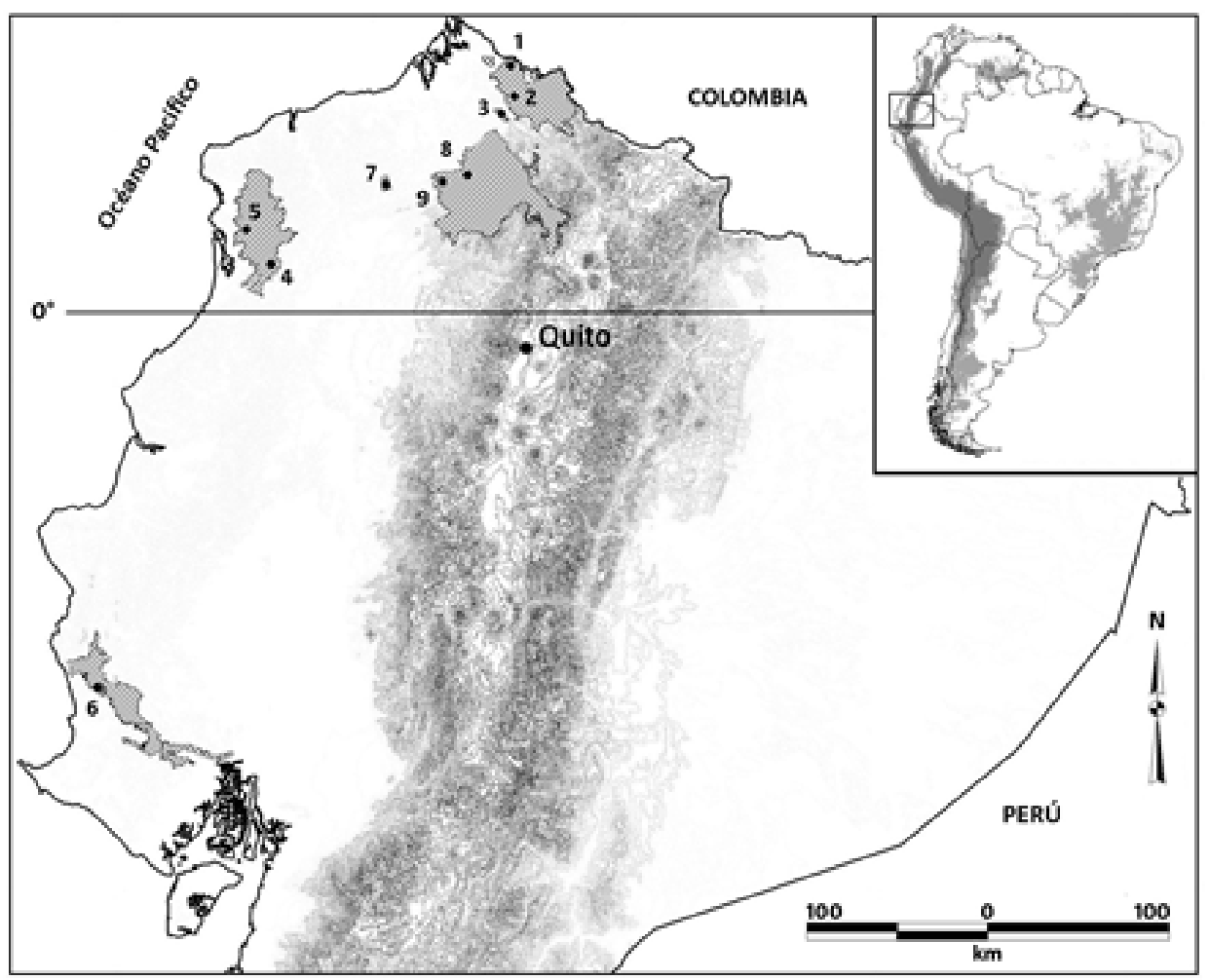

Figura 1. Mapa de Ecuador occidental. Se incluyen las cuatro áreas de estudio. Los puntos negros indican las localidades de muestreo: 1. Mataje, 2. Pambilar y 3. Río Bogotá en la Reserva Étnica Awá; 4. Estación Biológica Bilsa y 5. San Salvador en la Reserva Ecológica Mache-Chindul; 6. Loma Alta en el Bosque Protector Chongón-Colonche; 7. Refugio de Vida Silvestre El Pambilar, 8. Playa de Oro, 9. Estero Vicente en la Reserva Ecológica CotacachiCayapas y su área de amortiguamiento.

Tabla 1. Áreas de estudio y localidades de muestreo de pecaríes de labio blanco y jaguares en el Ecuador Occidental $^{*}$

\begin{tabular}{|c|c|c|c|c|c|c|}
\hline $\begin{array}{l}\text { Áreas de estudio/ } \\
\text { Localidades de muestreo }\end{array}$ & $\begin{array}{r}\text { Días de } \\
\text { muestreo }\end{array}$ & $\begin{array}{l}\text { Entrevistas } \\
\qquad(n)\end{array}$ & $\begin{array}{c}\text { Cámaras } \\
\text { (trampas/noche) }\end{array}$ & $\begin{array}{l}\text { Muestreos de } \\
\text { reconocimiento } \\
\qquad(\mathrm{km})\end{array}$ & Pecarí & Jaguar \\
\hline \multicolumn{7}{|l|}{ i. Reserva Étnica Awá (1100 km²) } \\
\hline 1. Centro Awá Mataje & 31 & 30 & 900 & 460 & No & No \\
\hline 2. Centro Awá Pambilar & 39 & 32 & 1029 & 350 & No & No \\
\hline 3. Centro Awá Río Bogotá & 31 & 20 & 505 & 300 & No & No \\
\hline \multicolumn{7}{|l|}{$\begin{array}{l}\text { ii. Reserva Ecológica Mache- } \\
\text { Chindul }\left(1200 \mathrm{~km}^{2}\right)\end{array}$} \\
\hline 4. Estación Biológica Bilsa & 29 & 12 & 600 & 199 & No & No \\
\hline 5. Comunidad Chachi San Salvador & 35 & 30 & 837 & 329 & No & No \\
\hline \multicolumn{7}{|l|}{$\begin{array}{l}\text { iii. Bosque Protecto Chongón- } \\
\text { Colonche }\left(700 \mathrm{~km}^{2}\right)\end{array}$} \\
\hline $\begin{array}{l}\text { 6. Comunidad Manteño- } \\
\text { Huancavilca Loma Alta }\end{array}$ & 34 & 35 & 829 & 250 & No & No \\
\hline \multicolumn{7}{|l|}{$\begin{array}{l}\text { iv. R.E. Cotacachi-Cayapas y área } \\
\text { de influencia }\left(2500 \mathrm{~km}^{2}\right)\end{array}$} \\
\hline $\begin{array}{l}\text { El Pambilar } \\
\text { 8. Comunidad Afroecuatoriana }\end{array}$ & 34 & 28 & 700 & 325 & Sí & Sí \\
\hline Playa de Oro & 39 & 30 & 950 & 546 & Sí & Sí \\
\hline 9. Comunidad Chachi Estero & & & & & & \\
\hline Vicente & 36 & 30 & 899 & 355 & Sí & Sí \\
\hline Esfuerzo de muestreo total & & 308 & 247 & 7249 & 3114 & \\
\hline
\end{tabular}

* Los muestreos se realizaron entre agosto de 2009 y agosto de 2011. Para cada localidad se indican los días de muestreo, el esfuerzo de muestreo realizado con los tres métodos utilizados y si las dos especies fueron registradas en la localidad 
muestreo, determinar las principales amenazas para la conservación, y la percepción de la gente sobre los cambios en la abundancia de ambas especies durante los últimos 10 años. Utilizamos además un promedio de 45 trampas fotográficas en cada localidad (CamTrakker equipadas con cámaras Canon), distanciadas al menos $500 \mathrm{~m}$ una de otra, en un área aproximada de $50 \mathrm{~km}^{2}$. En cada una de las estaciones de trampeo colocamos el perfume comercial Chanel $\mathrm{N}^{\circ} 5$ (http://www.chanel.com) como atrayente para felinos (Viscarra et al. 2011). El perfume se untó en un trozo de tela y dentro de una caja pequeña de plástico con agujeros para proteger la tela de la lluvia, y al mismo tiempo permitir que el aroma del perfume se dispersara en el ambiente. La independencia entre fotografías se basó en los parámetros utilizados por O'Brien et al. (2003): 1. Fotografías consecutivas de distintos individuos de la mismas especie, 2. Fotografías consecutivas de individuos de la misma especie tomadas con al menos 30 minutos de diferencia, y 3. Fotografías no consecutivas de individuos de la misma especie. Finalmente, los muestreos de reconocimiento implicaron el registro de observaciones directas e indirectas de fauna silvestre a lo largo de senderos preexistentes en el área de estudio.

Análisis de datos. El diseño de muestreo que utilizamos no nos permitió estimar la abundancia y densidad poblacional con el método tradicional de captura-recaptura. Sin embargo, utilizando las tasas de captura fotográficas, estimamos la densidad poblacional para ambas especies con el Modelo del Encuentro Aleatorio (MEA) propuesto por Rowcliffe et al. (2008):

$$
D^{\wedge}=y / t * \pi / v r(2+\theta)
$$

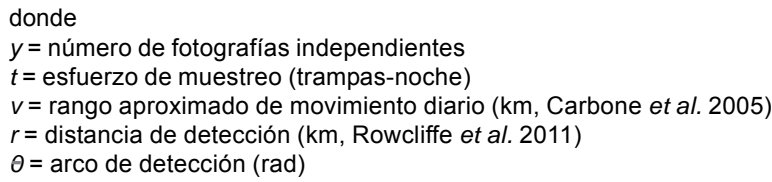

El modelo no requiere de la identificación de individuos para estimar la densidad de una población de fauna silvestre y se basa en el modelamiento de las tasas de encuentro de individuos que se asume mantienen movimientos aleatorios e independientes unos de otros, dentro de un espacio bidimensional (Carbone et al. 2001, Hutchinson y Waser 2007,
Rowcliffe et al. 2008, Rovero y Marshall 2009, Manzo et al. 2012, Zero et al. 2013). Los detalles operativos utilizados para realizar las estimaciones de densidad no se describen aquí porque existen publicaciones específicas para el efecto (e.g. Carbone et al. 2005, Rowcliffe et al. 2008, 2011, 2012).

\section{Resultados}

Durante 308 días de trabajo de campo en las nueve localidades de muestreo, realizamos 247 entrevistas, 7249 trampas/noche de trampeo fotográfico y $3114 \mathrm{~km}$ en senderos de reconocimiento. En seis de las nueve localidades no se registró ninguna de las especies buscadas, a pesar del importante esfuerzo de muestreo (Tabla 1). Estas seis localidades se encuentran en la Reserva Étnica Awá, la Reserva Ecológica Mache-Chindul y el Bosque Protector Chongón-Colonche. De acuerdo con las entrevistas, son más de cinco años desde que se observaron los últimos individuos de ambas especies $(83 \%$ de los entrevistados). Las personas entrevistadas consideran que las causas principales para la desaparición de las dos especies son la cacería (52\%) y la destrucción del hábitat (31\%). Otras causas, como la construcción de carreteras, explotación minera, la tala selectiva y cambios en el comportamiento de las especies representaron $17 \%$ restante de las respuestas.

En las otras tres localidades de muestreo, en la Reserva Ecológica Cotacachi-Cayapas y su área de amortiguamiento, sí se registraron pecaríes y jaguares (Tabla 1; Figura 2). Un total de 80 cazadores (91\% de los entrevistados) aseguraron haber visto pecaríes y $50(57 \%)$ aseguraron haber visto jaguares, durante los últimos cinco años. Los resultados de las entrevistas fueron congruentes con el trampeo fotográfico y los senderos de reconocimiento. Con las trampas fotográficas obtuvimos un total de 58 eventos independientes de pecarí (de un total de 76 fotografías) y nueve eventos independientes de jaguar (de un total de 10 fotografías). Con los muestreos de reconocimiento, por otra parte, realizamos un total de 37 registros de pecarí (dos de ellos observaciones directas) y cinco registros de huellas de jaguar.

Del total de cazadores entrevistados, $74 \%$ tiene la percepción de que las poblaciones de ambas especies están disminuyendo, y que ahora se ven con mucha menor frecuencia que hace 5 y 10 años atrás. 

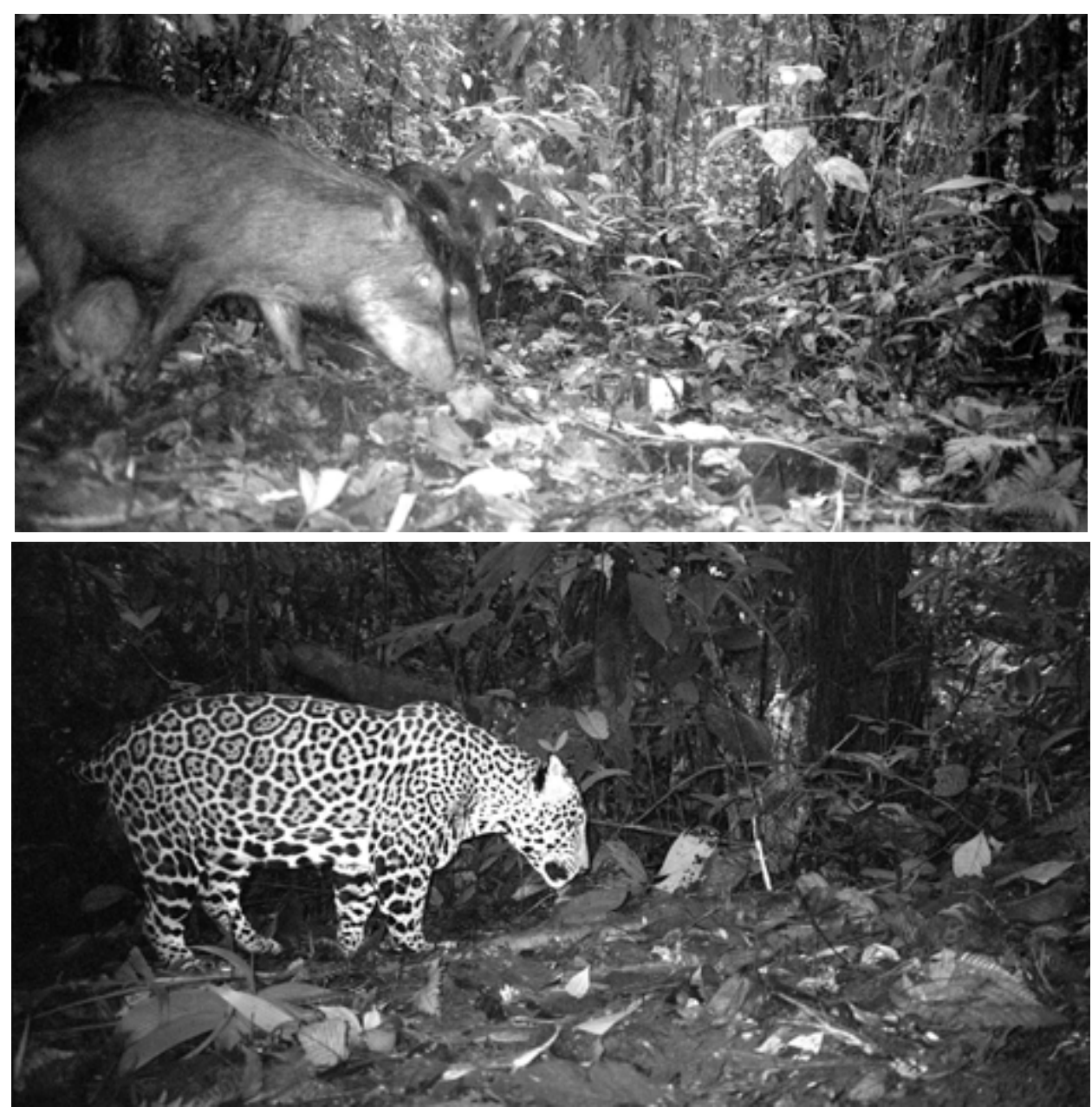

Figura 2. Pecaríes de labio blanco y jaguar fotografiados en la Reserva Ecológica Cotacachi-Cayapas y su área de amortiguamiento.

Las causas de esta reducción son atribuidas principalmente a la cacería (61\%) y a la construcción de carreteras (37\%). Hasta hace poco tiempo, en el área no existían carreteras, y el único acceso era por vía fluvial, a través de los ríos Santiago y Cayapas. Las personas entrevistadas aseguraron también que la cacería de ambas especies sucede periódicamente, sobre todo fuera de los límites de las áreas protegidas. Solo en los últimos cinco años se informó que se han cazado por lo menos cuatro jaguares por conflicto con la gente. Los motivos de este conflicto se originan por la percepción de que los jaguares son potencialmente peligrosos para los seres humanos, más que por la existencia de individuos problema que causan pérdidas en los animales domésticos.

Con base en el MEA, la densidad poblacional estimada del pecarí fue de 23,65 \pm 4,78 individuos/
$100 \mathrm{~km}^{2}\left(D^{\wedge} \pm\right.$ IC no paramétricos 95\%; $y=58 ; t=$ 2549 trampas-noche; $v=8,22 \mathrm{~km} ; r=0,0169 \mathrm{~km} ; \theta$ $=0,175 \mathrm{rad})$. La densidad poblacional del jaguar, en cambio, fue de 2,63 $\pm 1,96$ individuos $/ 100 \mathrm{~km}^{2}(y=$ 9; $t=2549$ trampas-noche; $v=9,57 \mathrm{~km} ; r=0,0202$ $\mathrm{km} ; \theta=0,175 \mathrm{rad})$. El remanente de $2500 \mathrm{~km}^{2}$ donde registramos los pecaríes y jaguares incluyó la parte baja de la Reserva Ecológica Cotacachi-Cayapas (por debajo de 2000 m), el Refugio de Vida Silvestre El Pambilar, y el área contigua a ambas (actualmente área no protegida y designada como Bloque $10 \mathrm{de}$ Patrimonio Forestal del Estado, Figura 3). Por otra parte, uno de los jaguares que fueron capturados fotográficamente en el Refugio de Vida Silvestre El Pambilar (040'15,2" N-7853'58,3" W; el 24 de abril de 2011) fue recapturado dos meses después a $32 \mathrm{~km}$ de distancia, en línea recta, en la Reserva 


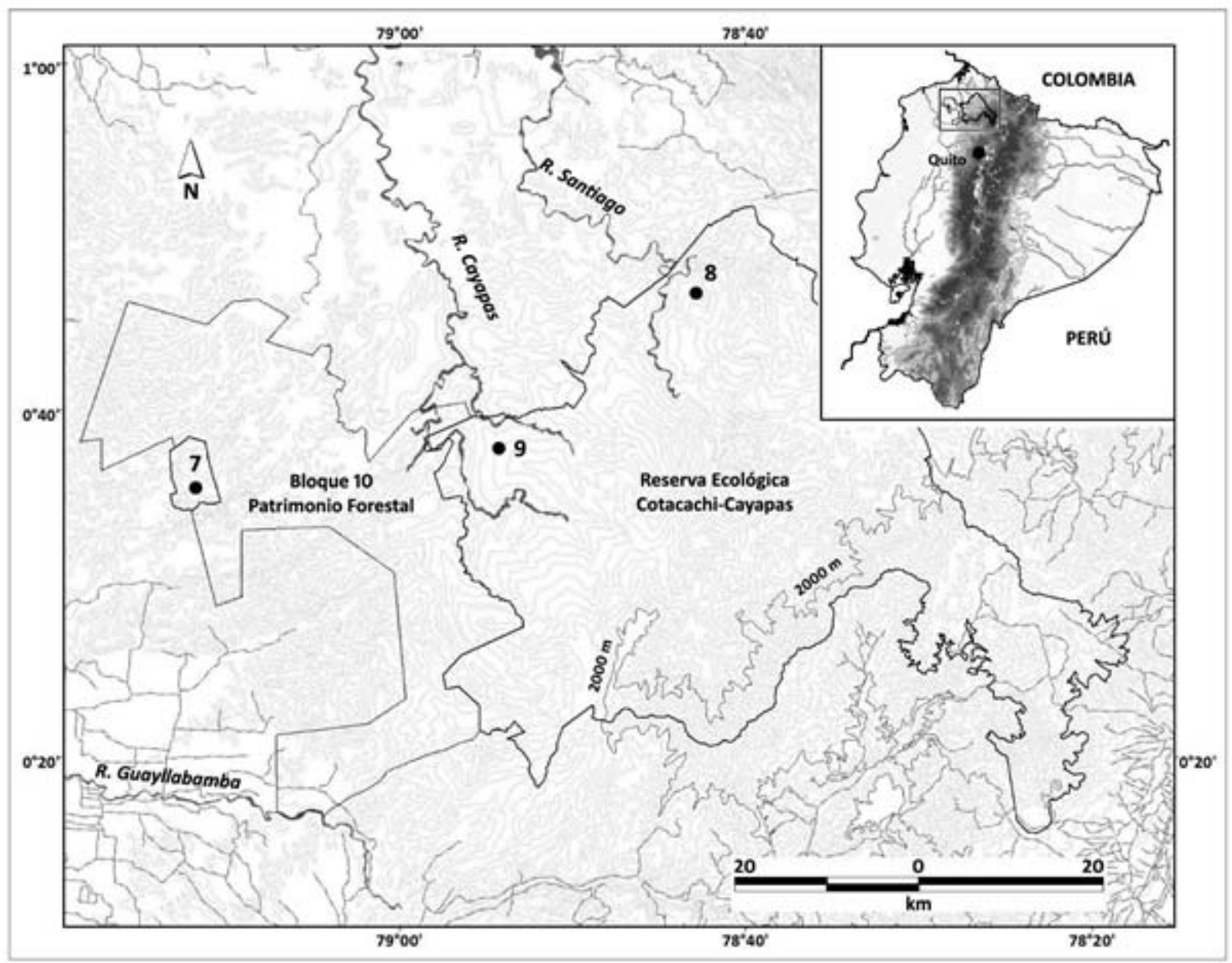

Figura 3. Mapa del remanente de bosque más grande $\left(2500 \mathrm{~km}^{2}\right)$ y mejor conservado del Ecuador occidental. Los números corresponden a las localidades de muestreo: 7. Refugio de Vida Silvestre Pambilar, 8. Playa de Oro, 9. Estero Vicente en la Reserva Ecológica Cotacachi-Cayapas. Se indica la curva de nivel de 2000 m, el límite altitudinal aproximado del ámbito de distribución para el pecarí de labio blanco y jaguar. Al sur de las tres áreas se puede observar una red de carreteras de segundo orden que son utilizadas para la extracción de madera.

Ecológica Cotacachi Cayapas $\left(0^{\circ} 36^{\prime} 47,2^{\prime \prime} \mathrm{N}-\right.$ 79¹0’51,6" W; el 22 de junio de 2011).

\section{Discusión}

Los métodos de muestreo de mamíferos grandes utilizados en este estudio (entrevistas a cazadores, trampas fotográficas y muestreos de reconocimiento) produjeron resultados congruentes entre sí. El conocimiento tradicional se ha valorado mucho en los últimos años, y las entrevistas a gente local representan un método de muestreo rápido y eficiente para evaluar la presencia-ausencia de fauna silvestre y sus tendencias poblacionales, al mismo tiempo que involucran directamente a la gente de las comunidades locales en los esfuerzos de conservación
(Hellier et al. 1999, Kimmerer 2000, Moller et al. 2004). El trampeo fotográfico es una herramienta efectiva para detectar especies raras y esquivas como los jaguares y los pecaríes (e.g. Wallace et al. 2003, Silver et al. 2004, Soisalo y Cavalcanti 2006). Aunque las trampas fotográficas se han utilizado principalmente para estimar abundancia a través de modelos de captura-recaptura, la misma técnica puede ser utilizada para estimar la densidad poblacional sin la necesidad de identificar individuos (Rowcliffe et al. 2008, Rovero y Marshall 2009, Manzo et al. 2012, Zero et al. 2013). En cuanto a los muestreos de reconocimiento, la mayor crítica a esta metodología es que la información obtenida tiene un sesgo por ser colectada a lo largo de senderos preexistentes. Sin embargo, en pruebas empíricas se ha demostrado que 
existe una fuerte correlación entre datos colectados en senderos de reconocimiento y en transectos en línea (Fay 1991, Hall et al. 1998a, Hall et al. 1998b, Blake et al. 2007).

Aunque no podemos asegurar con total certeza que tanto el pecarí de labio blanco como el jaguar se han extinguido a nivel local en la Reserva Étnica Awá, la Reserva Ecológica Mache-Chindul, y el Bosque Protector Chongón-Colonche, los datos sugieren fuertemente que ambas especies han sido extirpadas o existen en densidades sumamente bajas por lo que probablemente han perdido su funcionalidad ecológica (Dirzo y Miranda 1990, Terborgh et al. 1999, Redford y Feinsinger 2001). Como resultado de los niveles masivos de deforestación en la Costa ecuatoriana, los remanentes de bosque actualmente son muy pequeños y aislados $\left(<0,1 \mathrm{~km}^{2}\right)$ y muchas de las especies de fauna silvestre más sensibles han sido extirpadas. En este contexto, la parte baja de la Reserva Ecológica Cotacachi-Cayapas, El Refugio de Vida Silvestre El Pambilar y el Bloque 10 de Patrimonio Forestal representan el remanente de bosque mejor conservado y más extenso del Ecuador occidental y la sección meridional del Chocó Biogeográfico (Figura 3). Por otra parte, la recaptura fotográfica de un individuo de jaguar destaca la importancia del Bloque 10 de Patrimonio Forestal en términos de provisión de conectividad entre el Refugio de Vida Silvestre El Pambilar y la Reserva Ecológica Cotacachi-Cayapas. Es así que el valor de esta área para la conservación de la biodiversidad es único. Además de pecaríes y jaguares en el área, registramos también varias especies de fauna silvestre que en el Ecuador son consideradas en Peligro Crítico de extinción, y que tampoco se registraron en los otros tres remanentes de bosque (e.g. pavón grande, Crax rubra; guacamayo verde mayor, Ara ambigua; tinamú de Berlepsch, Crypturellus berlepschi; y mono araña de cabeza marrón, Ateles fusciceps; Granizo et al. 2002, Tirira 2011).

En el contexto socioeconómico actual es improbable que los jaguares y pecaríes del Ecuador occidental puedan persistir a largo plazo sin acciones de conservación apropiadas y oportunas. Estas acciones deben incluir el control de actividades ilegales dentro de las áreas protegidas (e.g. control de la cacería y tala selectiva); la implementación de programas de educación ambiental para reducir los niveles de conflicto e incrementar la apreciación de la importancia ecológica de estas especies para el mantenimiento de ecosistemas saludables; la implementación de programas participativos de monitoreo de fauna silvestre para detectar tendencias poblacionales negativas de jaguares, pecaríes y otras especies, sobre todo dentro de las áreas protegidas; e integrar estas y otras estrategias de manejo y conservación de fauna silvestre en los planes de desarrollo local y los planes de ordenamiento territorial. En conjunto, la implementación de estas acciones de conservación puede mantener y restaurar poblaciones viables de jaguar y pecarí de labio blanco, en coexistencia con las poblaciones humanas, como parte integral de los ecosistemas y los paisajes humanos en el Ecuador occidental.

\section{Agradecimientos}

Este proyecto de investigación se realizó gracias al apoyo de la Agencia de los Estados Unidos para el Desarrollo Internacional (USAID) y el Woodland Park Zoo. Agradecemos al Ministerio del Ambiente de Ecuador (MAE) por extendernos los permisos de investigación (MAE-DPE-2010-039 y MAE-DPE2011-0137) y a Santiago García, José Eduardo Narváez, Rosario Tene, Wellington Montenegro, Fernando Morcillo y Víctor Tacuri, funcionarios del MAE, por apoyar nuestro trabajo en el campo. Agradecemos también a Olindo Nastacuás, Presidente de la Federación de Centros Awá del Ecuador (FCAE); Egberto Tapuyo, Presidente de la Comunidad Chachi de San Salvador y a Geovanny Catuto, Presidente de la Comunidad Manteño-Huancavilca de Loma Alta por permitirnos trabajar dentro de sus territorios ancestrales. Los comentarios de Érika Cuellar, Alex Jiménez Ortega y dos revisores anónimos, mejoraron las versiones iniciales de este manuscrito de forma sustancial.

\section{Literatura citada}

Altrichter M, Taber A, Beck H, et al. 2011. Range-wide declines of a key Neotropical ecosystem architect, the Near Threatened white-lipped peccary Tayassu pecari. Oryx. 46: 87-98.

Beck H. 2006. A review of peccary-palm interactions and their ecological ramifications across the Neotropics. $J$ Mammal. 87: 519-30. 
Blake S, Strindberg S, Boudjan P, et al. 2007. Forest elephant crisis in the Congo Basin. PLOS Biol. 5: e111.

Brooks TM, Mittermeier RA, Mittermeier CG, et al. 2002. Habitat loss and extinction in the hotspots of biodiversity. Conserv Biol. 16: 909-23.

Carbone C, Christie S, Conforti K, et al. 2001. The use of photographic rates to estimate densities of tigers and other cryptic mammals. Anim Conserv. 4: 75-9.

Carbone C, Cowlishaw G, Isaac NJB, Rowcliffe JM. 2005. How far do animals go? determinants of day range in mammals. Am Nat. 165: 290-7.

Dinerstein E, Olson DM, Graham DJ, et al. 1995. A conservation assessment of the terrestrial ecoregions of Latin America and the Caribbean. Washington, DC: The World Bank.

Dirzo R, Mendoza E. 2007. Size-related differential seed predation in a heavily defaunated Neotropical rain forest. Biotropica. 39: 355-62.

Dirzo R, Miranda A. 1990. Contemporary Neotropical defaunation and forest structure, function, and diversity: a sequel to J.W. Terborgh. Conserv Biol. 4: 444-7.

Dodson CH, Gentry AH. 1991. Biological extinction in western Ecuador. Ann Mo Bot Gard. 78: 273-95.

Fay JM. 1991. An elephant (Loxodonta africana) survey using dung counts in the forests of the Central African Republic. J Trop Ecol. 7: 25-36.

Fragoso JMV. 2005. The role of trophic interactions in community initiation, maintenance and degradation. In: Burslem DFRP, Pinard MA, Hartley SE (Eds.). Biotic Interactions in the Tropics: their role in the maintenance of species diversity. Cambridge: Cambridge University Press. pp. 310-27.

Granizo T, Pacheco C, Rivadeneira MB, Guerrero M, Suárez L (eds.). 2002. Libro Rojo de las Aves del Ecuador. Quito: SIMBIOE, Conservación Internacional, EcoCiencia, Ministerio del Ambiente, UICN.

Hall J, Saltonstall K, Inogwabini B, Omari I. 1998a. Distribution, abundance and conservation status of Grauer's gorilla. Oryx. 32: 122-30.

Hall J, White L, Inogwabini B, et al. 1998b. A survey of gorillas (Gorilla gorilla graueri) and chimpanzees (Pan troglodytes schweinfurthi) in the Kahuzi-Biega National Park lowland sector and adjacent forest in eastern Democratic Republic of Congo. Int J Primatol. 19: 20735.

Hellier A, Newton AC, Ochoa-Gaona S. 1999. Use of indigenous knowledge for rapidly assessing trends in biodiversity: a case study from Chiapas, Mexico. Biodivers Conserv. 8: 869-89.

Hutchinson JMC, Waser PM. 2007. Use, misuse and extensions of «ideal gas» models of animal encounter. Biol Rev. 82: 335-59.

Jones CG, Lawton JH, Shachak M. 1994. Organisms as ecosystem engineers. Oikos. 69: 373-86.

Jorge MLSP, Galetti M, Ribeiro MC, Ferraz KMPMB. 2013. Mammal defaunation as surrogate of trophic cascades in a biodiversity hotspot. Biol Conserv. 163: 49-57.

Keuroghlian A, Eaton DP. 2008. Fruit availability and peccary frugivory in an isolated Atlantic forest fragment: effects on peccary ranging behavior and habitat use. Biotropica. 40: 62-70.

Keuroghlian A, Eaton DP. 2009. Removal of palms fruits and ecosystem engineering in palms stands by white-lipped peccaries (Tayassu pecari) and other frugivores in an isolated Atlantic Forest fragment. Biodivers Conserv. 18: 1733-50.

Kimmerer RW. 2000. Native knowledge for native ecosystems. J Forest. 98: 4-9.

Manzo E, Bartolommei P, Rowcliffe JM, Cozzolino R. 2012. Estimation of population density of European pine marten in central Italy using camera trapping. Acta Theriol. 57: 165-72.

Mittermeier RA, Myers N, Thomsen JB, da Fonseca GAB, Olivieri S. 1998. Biodiversity hotspots and major tropical wilderness areas: approaches to setting conservation priorities. Conserv Biol. 12: 516-20.

Moller H, Berkes F, O’Brian Lyver P, Kislalioglu M. 2004. Combining science and traditional ecological knowledge: monitoring populations for co-management. Ecol Soc. 9: 2.

Myers N. 1988. Threatened biotas: hot-spots in tropical forests. Environmentalist. 8: 1-20.

Myers N, Mittermeier RA, Mittermeier CG, da Fonseca GAB, Kent J. 2000. Biodiversity hotspots for conservation priorities. Nature. 403: 853-8.

O’Brien TG, Kinnaird MF, Wibisono HT. 2003. Crouching tigers, hidden prey: Sumatran tiger and prey populations in a tropical forest landscape. Anim Conserv. 6: 131-9.

Painter RLE, Rumiz DI. 1999 ¿Por qué son los herbívoros terrestres importantes para los bosques de producción forestal? Rev Boliv Ecol Conserv. 5:61-74.

Parker III TA, Carr JL. 1992. Status of forest remnants in the cordillera de la costa and adjacent areas of southwestern Ecuador. Washington DC: Conservation International.

Redford KH, Feinsinger KH. 2001. The half-empty forest: sustainable use and the ecology of interactions. In: Reynolds JD, Mace GM, Redford KH, Robinson JG (eds.). Conservation of Exploited Species. Cambridge: Cambridge University Press. pp 370-99.

Rovero F, Marshall AF. 2009. Camera trapping photographic rate as an index of density in forest ungulates. $J$ Appl Ecol. 46: 1011-7.

Rowcliffe JM, Field J, Turvey ST, Carbone C. 2008. Estimating animal density using camera traps without the need for individual recognition. J Appl Ecol. 45: 1228-36.

Rowcliffe JM, Carbone C, Jansen PA, Kays R, Kranstauber B. 2011. Quantifying the sensitivity of camera traps: an adapted distance sampling approach. Methods Ecol Evol. 2: 464-76.

Rowcliffe JM, Carbone C, Kays R, Kranstauber B, Jansen PA. 2012. Bias in estimating animal travel distance: the effect of sampling frequency. Methods Ecol Evol. 3: 653-62.

Sanderson EW, Redford KH, Chetkiewicz CB, Medellín RA, Rabinowitz AR, Robinson JG, Taber AB. 2002. Planning to save a species: the jaguar as a model. Conserv Biol. 16: $58-72$.

Sierra R. 1999a. Traditional resource-use systems and tropical 
deforestation in a multi-ethnic region in north-west Ecuador. Environ Conserv. 26: 136-45.

Sierra R. 1999b. Propuesta preliminar de un sistema de clasificación de vegetación para el Ecuador continental. Quito: EcoCiencia, Ministerio del Ambiente.

Sierra R, Campos F, Chamberlin J. 2002. Assessing biodiversity conservation priorities: ecosystem risk and representativeness in continental Ecuador. Landscape Urban Plan. 59: 95-110.

Sierra R, Stallings J. 1998. The dynamics and social organization of tropical deforestation in Northwest Ecuador, 19831995. Hum Ecol. 26: 135-61.

Silver SC, Ostro LET, March LK, et al. 2004. The use of camera traps for estimating jaguar Panthera onca abundance and density using capture/recapture analysis. Oryx. 38: 14854.

Soisalo MK, Cavalcanti SMC. 2006. Estimating the density of a jaguar population in the Brazilian Pantanal using camera-traps and capture-recapture sampling in combination with GPS radio-telemetry. Biol Conserv. 129: 487-96.

Taber A, Chalukian SC, Altrichter M, et al. 2008. El destino de los arquitectos de los bosques neotropicales: evaluación de la distribución y el estado de conservación de los pecaríes labiados y los tapires de tierras bajas. New York:
Wildlife Conservation Society, Tapir Specialist Group, Wildlife Trust.

Terborgh JW. 1988. The big things that run the world: a sequel to E.O. Wilson. Conserv Biol. 2: 402-3.

Terborgh JW, Estes JA, Paquet P, et al. 1999. The role of top carnivores in regulating terrestrial ecosystems. In: Soulé ME, Terborgh JW (eds.). Continental conservation: scientific foundations of regional reserve networks. Washington DC: Island Press. pp. 39-64.

Tirira D. 2007. Mamíferos del Ecuador: guía de campo. Quito: Ediciones Murciélago Blanco.

Tirira D. 2011. Libro Rojo de los Mamíferos del Ecuador. Quito: Fundación Mamíferos y Conservación, Pontificia Universidad Católica del Ecuador, Ministerio del Ambiente.

Viscarra ME, Ayala G, Wallace R, Nallar R. 2011. The use of commercial perfumes for studying jaguars. Cat News. 54: $30-1$.

Wallace RB, Gómez H, Ayala G, Espinoza F. 2003. Camera trapping for jaguar (Panthera onca) in the Tuichi valley, Bolivia. Mastozool Neotrop. 10: 133-9.

Zero VH, Sundaresan SR, O'Brien TG, Kinnaird MF. 2013. Monitoring an endangered savannah ungulate, Grevy's zebra Equus grevyi: choosing a method for estimating population densities. Oryx. 47: 410-9. 